\title{
DESIGN, ANALYSIS AND DEVELOPMENT OF SPECIAL PURPOSE MACHINE TO CARRY HEAVY LOADED COMPONENTS
}

\author{
Vedvyas $^{1}$, Naveen Bharadishettar ${ }^{2}$ \\ ${ }^{1} P G$ Student, Department of Mechanical Engineering, MVJ College of Engineering, Bengaluru, India \\ ${ }^{2}$ Assistant Professor, Department of Mechanical Engineering, MVJ College of Engineering, Bengaluru, India
}

\begin{abstract}
In the olden days very complicated thing is in the industry is to carry heavy loaded components and lifting from one place to another place, human effort needs lots of energy and it takes more time for same work, there are many huge and large amount of load lifting machine available, in that first started with gantry hoist and gantry cranes with which they carry heavy loads from one place to other, it works so far by the time but it can operate only in open place, in small scale industries it can't work, during the period the need is new machine which works in small scale industries with good time, the changing the generation with the new technology industries are planned to develop the special purpose machine which is used to lift the heavy loads. Structural analysis of the "Gantry Structure" has been carried out to find the mechanical response of the structure, subjected to applied loads and boundary conditions. The results include deformed shape of the structure, displacements at required locations. Modal analysis done for checking the model, Gravity Loading Applied for different position and checked for the deformation of Solid Beam for different position. Optimization is done to reduce the cost of the structure. The response of the structure got by structural analysis using MSC Nastran.
\end{abstract}

Keywords: Gantry, Types of gantry, Structural analysis, optimization, modal analysis, HyperMesh, NASTRAN.

\section{INTRODUCTION}

With vigorous development in the industries like marine engineering equipment, ship building, aerospace and other manufacturing industries the processing demand of large and complex parts increasingly daily. Now days most of the manufacturing industries are associated with heavy machineries and equipment to lead the advance technology, as such "gantry machine" a kind of load lifting equipment is widely using in most of the industries. $R \& D$ of the gantry machine tool has been taken an important study that includes design, analysis and optimization of the entire gantry structure. Gantry structure is kind of heavy loading lifting machine from one place to another place it benefits to accomplish work very effectively and efficiently withoptimized time and reduction in the human work.

When the technology improved with that value of time as a component of cost saving, the structure should be optimized by means of redesigning it by reducing the material hence, optimization. In gantry structure, beam plays a very important role in dynamic and static characteristics. As in the present study behavior of the beam is studied by considering different types of load.

Some of the research paper explains about that strength and stiffness of gantry machine and explained about middle place of the beam having maximum strength in its internal structure.Liand and Lio have worked on the FEM analysis of crossbeam by varying rib plates. Later Shi conducted modal analysis and got weakness in cross beam to do further optimization. The first resonance frequency and work space using the optimization based on functional dependency and geometry. gantry machine which having large work space for accessible by compared to many machines, so they told that using optimization they got kinematic design and gives the work space and frequency more than $50 \mathrm{hz}$ They studied on structural analysis of gantry CNC machine how much strength that gantry beam will withstand and got the deformation as well as stress distribution of beam.

Some studies explained about finite element and modal analysis theory for better improvement of performance as well as structure of machine with static and dynamic analysis and predicting optimal design for optimization. With reference to literature study present research focused on the static and modal analysis of gantry structure presented the stress levels and displacement. The final results are used to optimize the entire structure which lead to reduce cost of the whole machine.

Optimization requires mainly includes shape optimization, size optimization and topological optimization. Structural optimization is high level in topological optimization; topology information is carried out here. Wang' in Dalian university he carried out the topological optimization on a cross beam in gantry machine and finally $6 \%$ lighter than original, then Jun Xin Liu and Zhi Dong Li used guideweight method to solve topological optimization by having multiple loads. Traditional design replaced by structure optimization with goal oriented systematic method, the main thing is to see the lower the cost with best performance of structure using topology optimization it's also known as structural layout [5]. 
Gantry beam it is part of full machine to which static analysis given on the stress position using FE analysis , without changing geometry size optimized to it so gantry beam stiffness and strength increased, to improve reliability of that beam strengthening ribs added to it gantry beam quality reduced and optimized the gantry[6].

\section{PROPOSED WORK}

Based on the literature study, the present work mainly focused on the following objectives:

- Newly developed Special purpose machine is associated with modern technology.

- Proposed design completes the task very easily and accurately.

- Completely self and sufficiently working within the required time.
- Fastest task completing capability, accessibility for operation and maintenance.

\section{DESIGN AND DEVELOPMENT}

The earlier industries existing design are quite versatile, here designed model based on existing models only but differs in materials properties and strength of the structure varies, in this project expected to have a good capability of lifting the weights with accuracy and mobility must maintained. The earlier design structures are huge with that expenses of manufacturing also too high, so in this re designed model which is optimized to save the cost and expected effective work operation within required time.

\subsection{CAD Model}

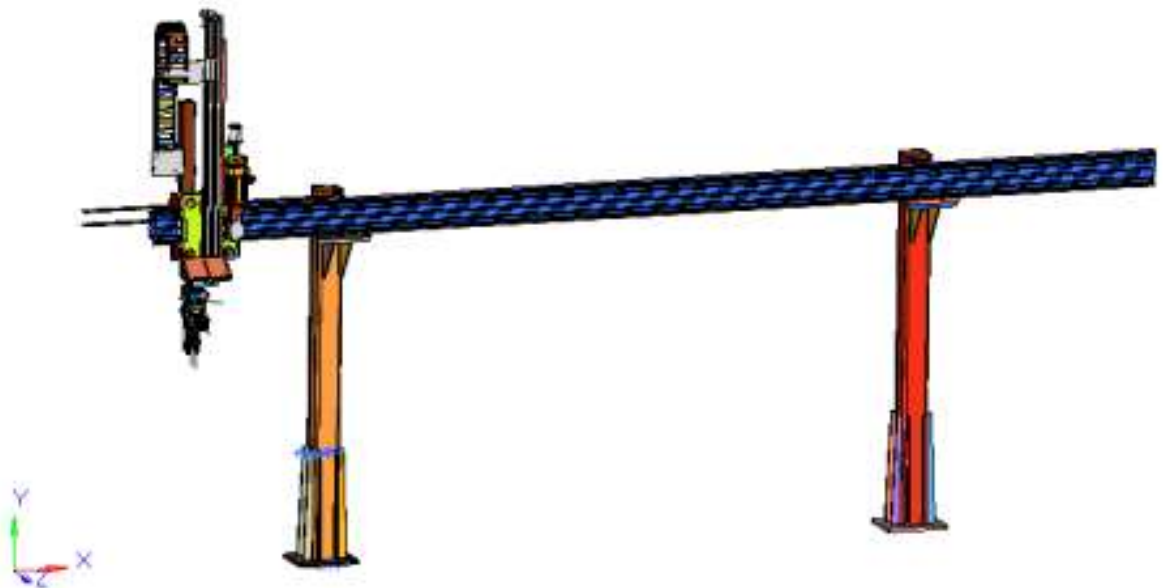

Fig. 1: Cad Model Gantry

\subsection{Design Calculations}

Loading Condition: Gravity Loading Applied for different position and check for the deformation of Solid Beam for different position.

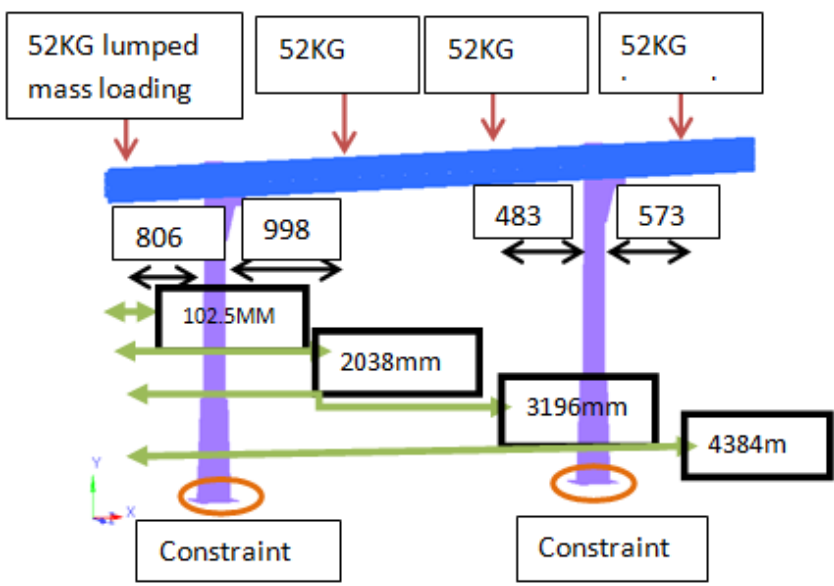

Fig.2: Loading condition

The beam which is simply supported with varying loads which is free to rotate, the reaction forces $\mathrm{R} 1$ and $\mathrm{R} 2$ are not known. To find the R1\&R2

$$
\begin{gathered}
\mathrm{R}_{1}+\mathrm{R}_{2}=0 \\
R 1=\frac{510.12 \times 872}{2659}=168.18 \mathrm{~N} \\
R 1=\frac{510}{872} \\
510.12 \times 872+\frac{R 2}{2779}=0 \\
R 2=510.12 \times \frac{872}{2779} \\
\mathrm{R}_{2}=-160.06 \mathrm{~N}(\text { compressive }) \\
\text { Bending Stress }==\sigma=\frac{\mathrm{MZ}}{\mathrm{I}} \\
=\frac{54397 \times 77}{3900000} \\
=6.25 \mathrm{MPa} \quad=0
\end{gathered}
$$

Average shear stress $=\frac{S}{A} x$ FOS

$=3.80 \times 10^{-5} \mathrm{MPa}$ 
HAND CALCULATIONS WITH SHEAR FORCE AND BENDING MOMENT

(Load case 01)

Calculating reaction force: $R_{1}+R_{2}=0$

$510.12 \star 872+R_{2} \star 2779=0$
$R_{1}=510.12 * 872 / 2659=167 ! 18 \mathrm{~N}$

$\left(\mathbf{R}_{1}: 510 \star 872\right)$

$R_{2}=510.12 * 872 / 2779$

$R_{2}=-160.06 \mathrm{~N}$ (compressive)

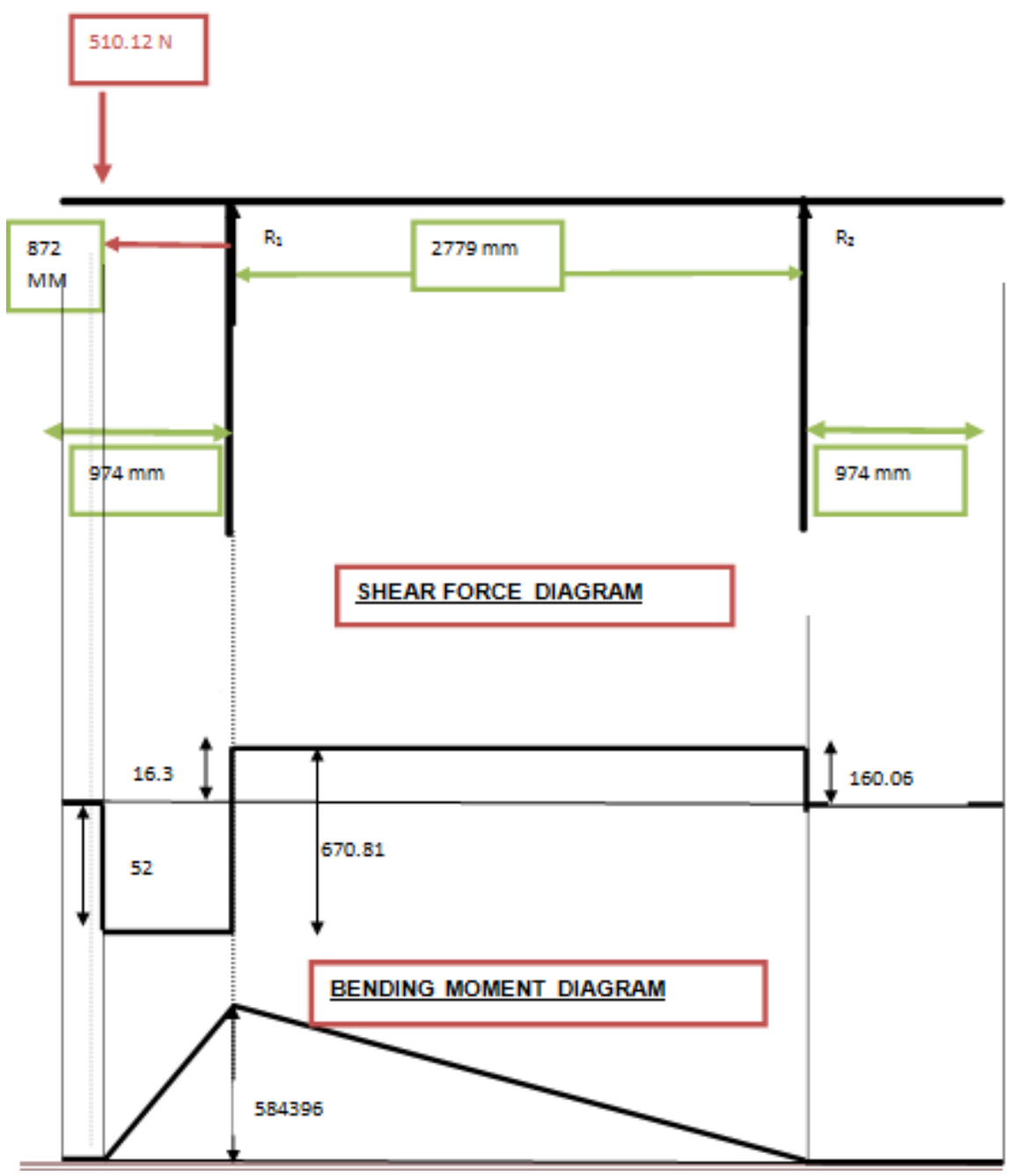

Fig.3: SFD and BMD Diagram

\section{ANALYSIS.}

FEM subdivides a large problem into smaller, simpler, parts, called finite elements. The simple equations that model these finite elements are then assembled into a larger system of equations that models the entire problem.

\subsection{FEM Model}

Hyper mesh is a FE pre-processor and post processor for the FE solver, design which is analyzed by engineer with the high effective performed and visual environment. It is easy to understand and learn with support direct to use the CAD geometry whichprovides good efficiency. It allow to optimize meshes from a criteria change meshes using morphing and create mid surface by the model which vary in thickness.

\subsection{Nastran Procedure To Solve Structural}

\section{Problem.}

1. Geometry- Import the CAD model from hyper mesh

2. FE Modeling

3. Checking the model

4. Analyzing model

5. Post processing

6. Document Results 


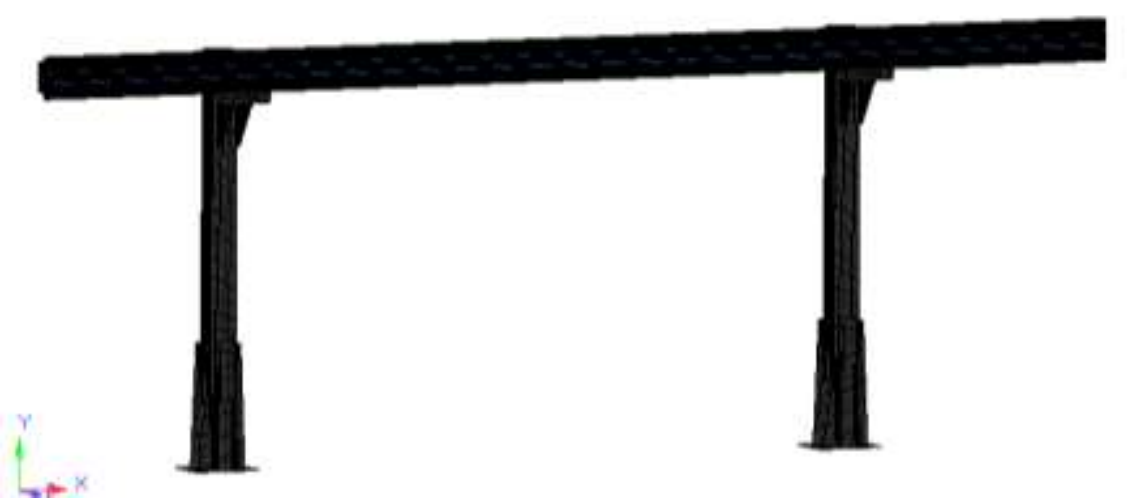

Fig-4: FE Model of gantry

\section{RESULTS AND DISCUSSION}

\subsection{Structural Analysis}

Structural analysis which determine the effect of loads on structure as well as their components. The structure mainly subjected to the analysis must withstand loads for Example Bridge, vehicle, furniture, biological tissue. Structural analysis used to show the fitness of thing tested. Which saves the physical test it is the most important part in engineering to know design of structure, it mainly incorporate field of applied math's, material science to compute deformation of structure, forces of internal and stress, reaction which support to it stability. In structural analysis mainly see the Structure and Load .Structure which refers as body used to support the load in civil we use to observe, in other branches ship and aircraft frames, pressure vessel, mechanical system. It is for safety purpose to do this test and an engineer must see the serviceability and safety. Total maximum stress: $8.6 \mathrm{mpa}$

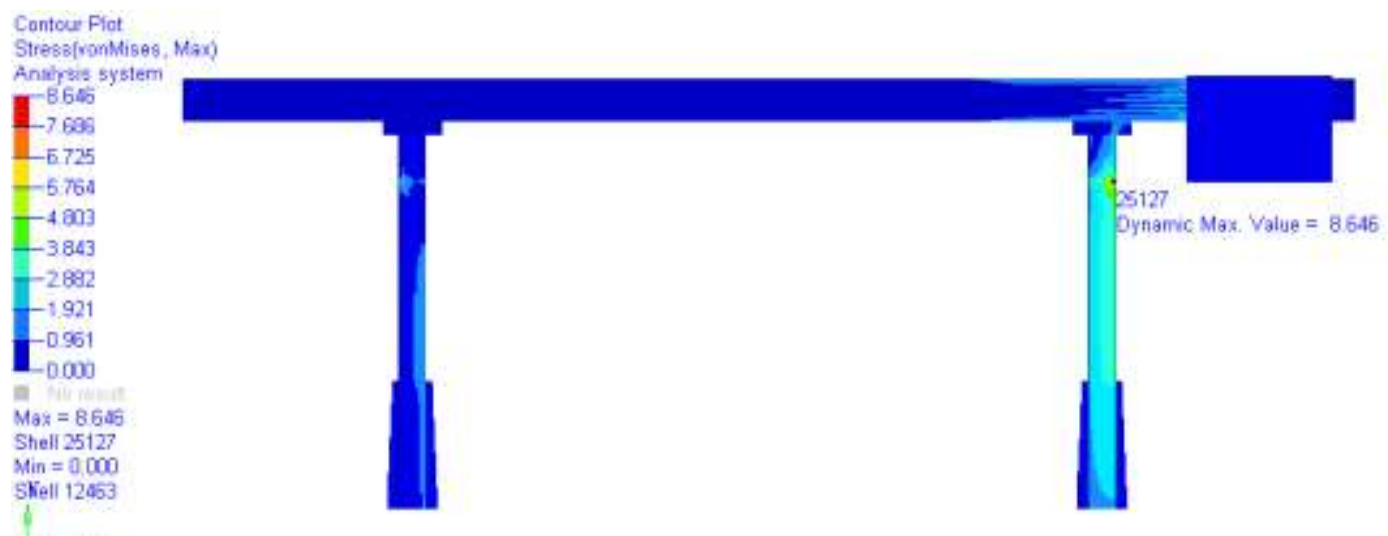

Fig. 5: Maximum Stress

Maximum shear stress: $3.891 \mathrm{MPa}$

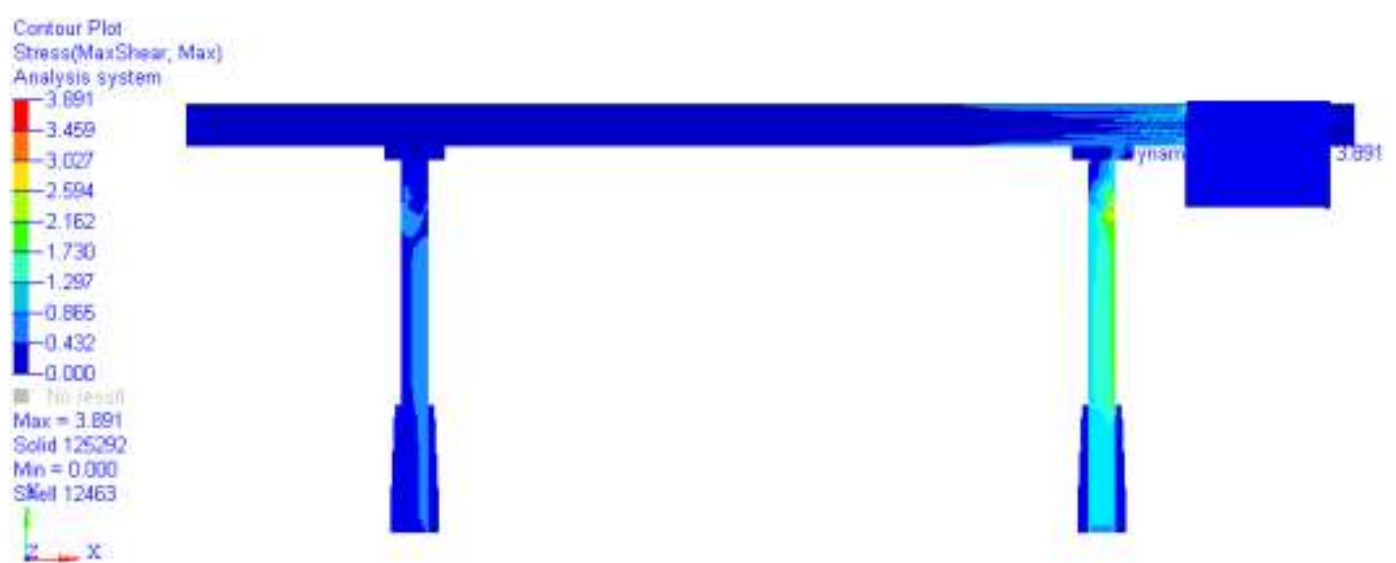

Fig.6: Maximum shear stress

Total Maximum Displacement $=0.817 \mathrm{~mm}$ 


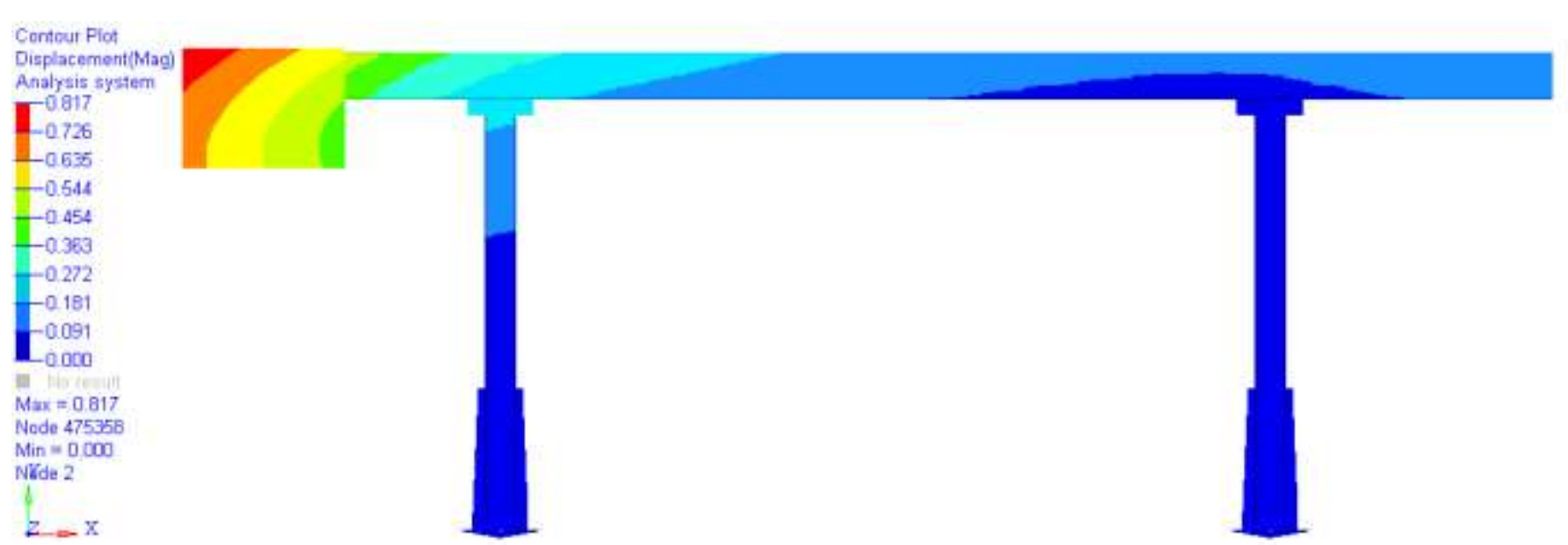

Fig. 7: Maximum Displacement

\subsection{Optimization}

Optimization means add or remove of material for the cost effective as well as how engineer needs that by that stresses may vary definitely with that strength and stiffness too. So it depends on system how should operate the thing reduce or increase.
1. Constrained optimization problem: which related to one or more constraints.

2. Unconstrained optimization problems: no constraint in this.

Max Displacement: 1.179MM

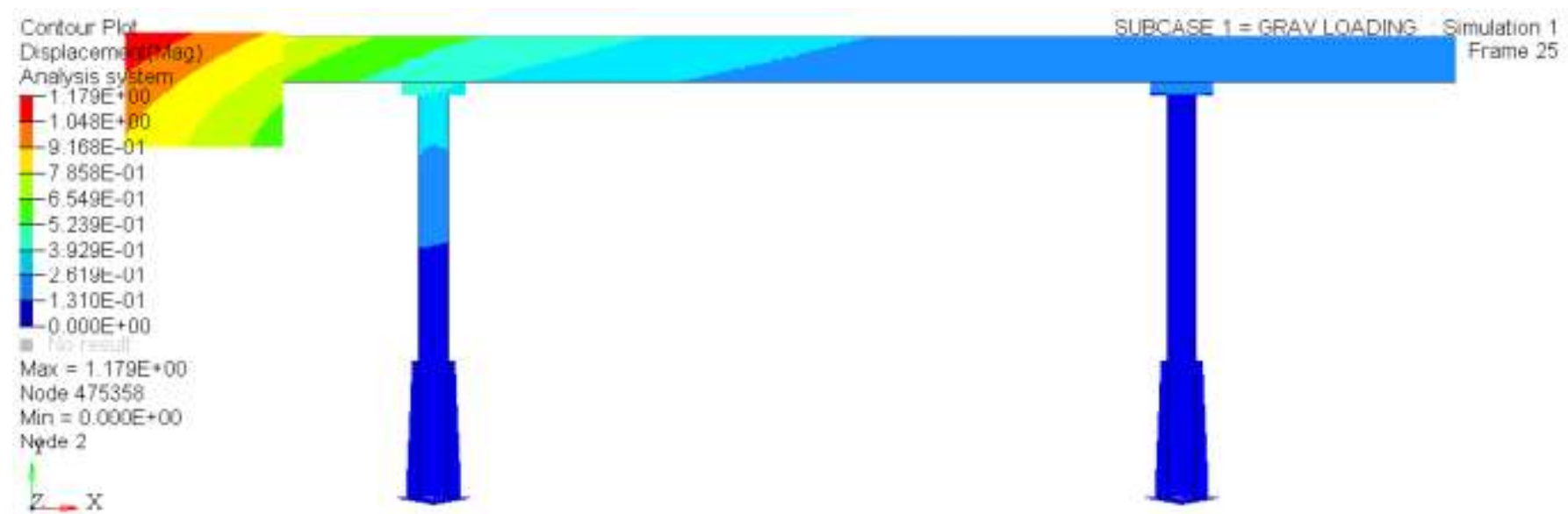

Fig. 8: Max displacement

Total maximum stress: $23.49 \mathrm{MPa}$

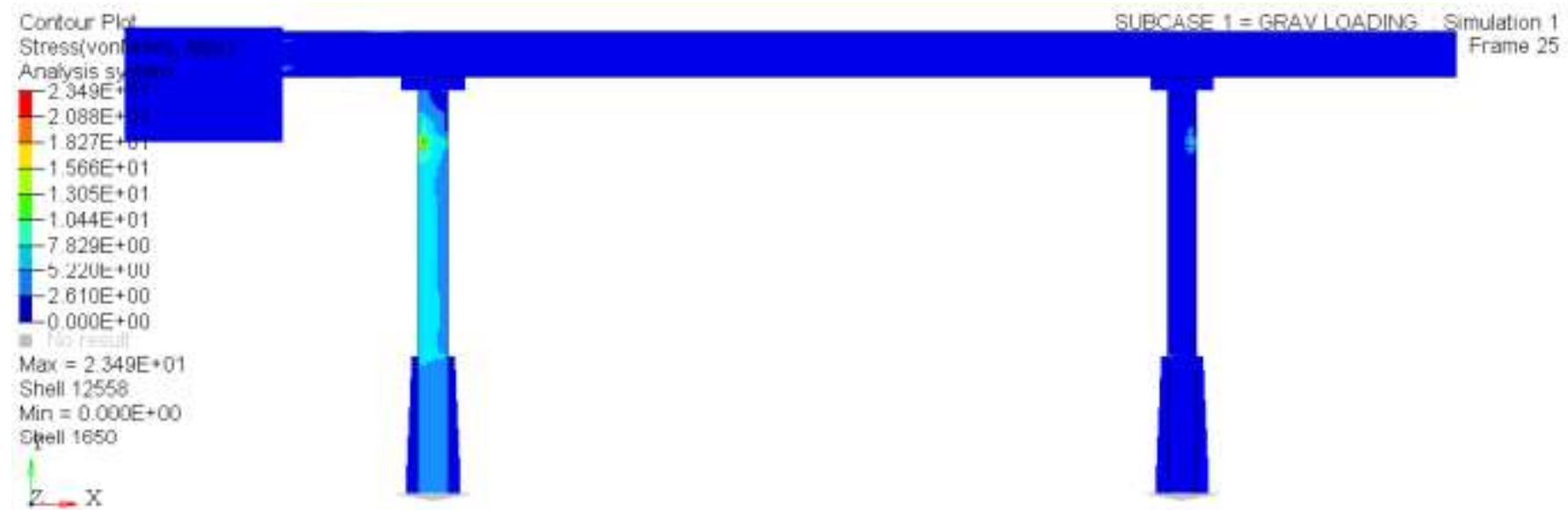

Fig. 9: Maximum Stress

Maximum shear stress: $8.53 \mathrm{MPa}$ 


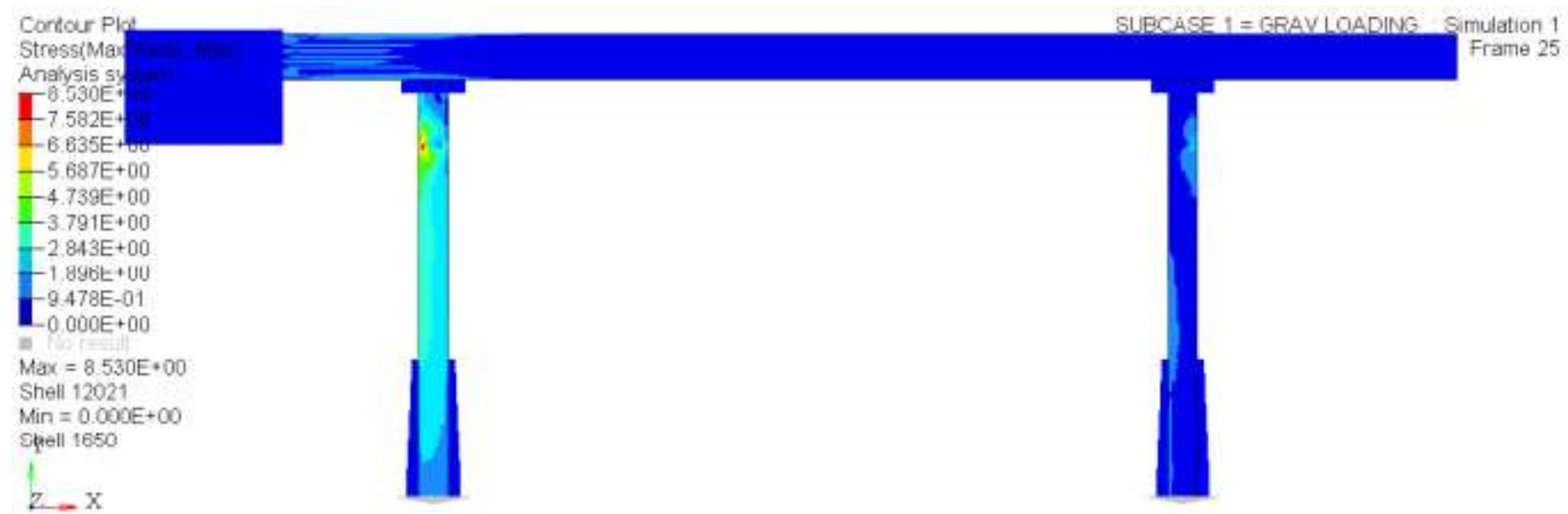

Fig. 10: Maximum Shear Stress

\subsection{Modal Analysis}

MODES (RESONANCE) are inherent properties of structure;it is determined by mainly the properties those are mass, stiffness, damping properties). Natural frequency defined to mode, modal damping and mode shape. If boundary condition of structure changed than modes also change Consider that mass added to a pump vertical it vibrates because mode changed. Natural frequency of a mode the overall operating deflection shape of the machine will dominate by mode shape of resonance. Operating deflection shape means on structure any forced motion that to of two or more points.

Frequency $=103.3 \mathrm{~Hz}$

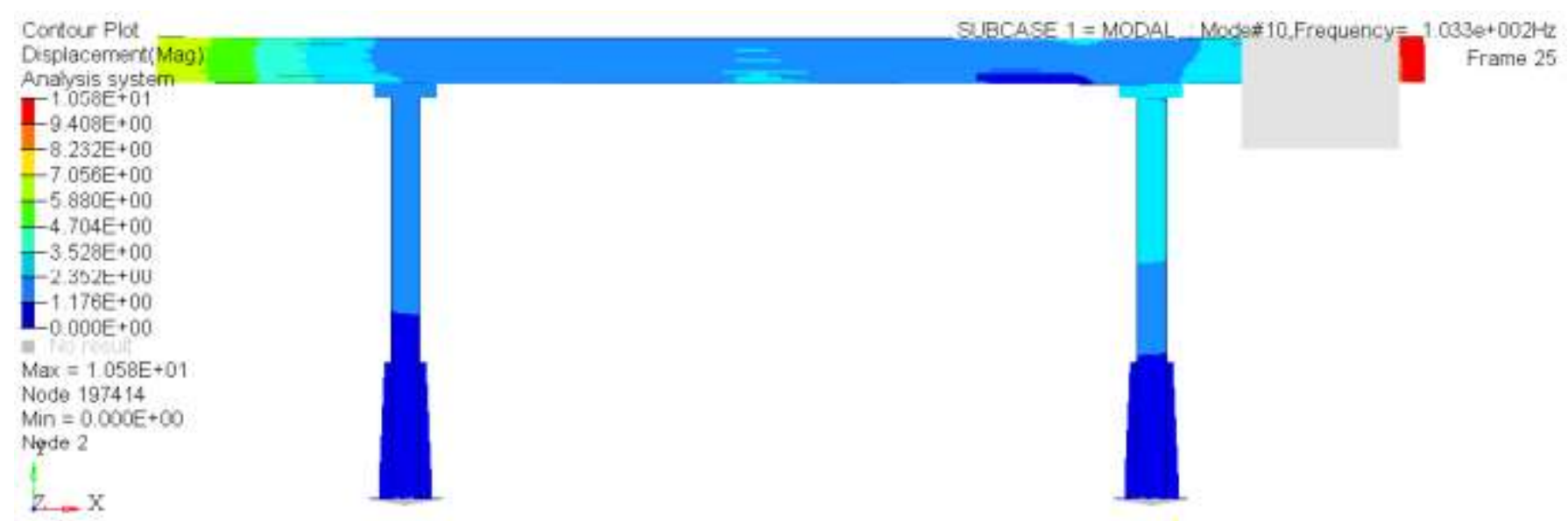

Fig-11: Mode Shapes

\section{CONCLUSION}

Gantry structure completely Analyzed using fem software, the beam which is under the load subjected to the structural analysis the hand calculation are matches with FEA results, the stress of FEA is $8.646 \mathrm{Mpa}$ and shear is $3.891 \mathrm{Mpa}$ with that displacement $0.559 \mathrm{~mm}$ this is of before optimization result Fig-(4,5,6). The actual design results which shows the gravity load of that beam, how much it can withstand the load of the respective acting weight. The result this is of maximum out of four loading condition the right end of the gantry which having highest stress, so that considering optimized the gantry structure the stress got in this is maximum stress is $23.49 \mathrm{Mpa}$ and shear is $7.79 \mathrm{Mpa}$ ,displacement $0.890 \mathrm{~mm}$ Fig-(7,8,9) respectively showed.

The results compared stress is increased because of reducing the thickness of the column of gantry structure, after optimization the stress is doubled compared to actual design with displacement. To the model modal analysis is carried out which having the frequency of $103.3 \mathrm{~Hz}$. The strength and stiffness of material which is maintained high itself by varying the thickness.

\section{REFERENCES}

[1]. X.wang , j.zia, f yang, and s.jhang, "The topological optimation design and analysis for gantry machine tool cross beam component" manufacturing technology machine vol.59, no.11, pp. 64-68, 2009

[2]. B. li, z. Fang, and q. Liang, "deformation analysis and optimization for the beam rails of the fix beam gantry machine centre" electrical engineering technology, vol. 40, no .11, pp. 104-145, 2010.

[3]. Y. guan, D. Mu, Y. Zhao, and Larne, "finite element analysis of GMCU2060 Gantry machine centre crossbeam," machine tool hydraulics, vol.39, no.11. pp. 131-134,2011 
[4]. L.Han, and K. saan, "anti-deformation processing gantry rail base and the excel based COSMOWORKS," world manufacturing engineering \& market, vol. 25, no.3, pp. 96-98, 2012

[5]. Li qilang, go xuhong, cheyao, jiwenzhng, wangling and jhangtao, "mechanical analysis of crossbeam in a gantry machine tool and its deformation compensation. College of mechanical engineering .sooachowuniversity,Jiaangsu 215006 ,p.r.china.Vol. 9,pp .213-218,2015

[6]. "stress analysis and optimization design of gantry beam". By wu li-wng, li Yao fi, yang she longa, wangze kai. Vol 543-547(2014)pp 50-54.

[7]. Montogomry, d.c. design and analysis of experiments, wiley: newyork,pp 181-13,2001.

[8]. Rao, s.s, engineering optimization: theory and practice, john wiley\&sons: newyork, pp,436-442,1996.

[9]. Y. Li, L.jhang, c.liu and, g. yine," static rigidity analysis and structure optimization of five axis gantry type machining center "modular machine tool automatic manufacturing technique,vol.53, no.6, pp. 9-16,2011

[10]. "Structural optimization of the cross beam of a gantry machine tool based on grey relational analysis by shihaooliu. Yue li, yulanlio. Zhizhongaguo 21 December 2013. 